\title{
The Effective Part of Dioscorea Nipponica Makino on Esophageal Carcinoma Cells and the Interaction with Human Serum Albumin
}

\author{
Fuchun SI ${ }^{1, \mathrm{a}, *}$ \\ ${ }^{1}$ Laboratory of molecular biology for TCM \\ Henan University of Chinese Medicine \\ Zhengzhou 450046, China \\ a e-mail: sifc2000@ hotmail.com
}

\author{
Wenbin $\mathrm{WANG}^{1,2, \mathrm{~b}}$ \\ ${ }^{1}$ Laboratory of molecular biology for TCM \\ Henan University of Chinese Medicine \\ Zhengzhou 450046, China \\ ${ }^{2}$ National Key Laboratory of Cryospheric Sciences/Tien \\ Shan Glaciological Station, Cold and Arid Regions \\ Environmental and Engineering Research Institute \\ Chinese Academy of Sciences \\ Lanzhou 730000, China \\ b e-mail: wwellbin@126.com
}

\begin{abstract}
The effective part (BESDM) was extracted from the Dioscorea nipponica Makino. Cytotoxicity of the BESDM on Ec9706, Eca109 and TE1 cells tested by MTT assay. The result showed the Ic50 values of Ec9706, Eca109 and TE1 are $7.87 \pm 0.08, \quad 3.76 \pm 0.04$ and $13.53 \pm 0.06 \mu \mathrm{g} \cdot \mathrm{ml}^{-1}$, respectively. Fluorescence was used to study the interaction of BESDM with human serum albumin (HSA). The intrinsic fluorescence of HSA was quenched by BESDM. The quenching mechanism is mainly static quenching. The thermodynamic parameters, $\Delta H(-1656.79$ $\left.\mathrm{kJ} \cdot \mathrm{mol}^{-1}\right), \Delta \mathrm{S}\left(-5381.62 \mathrm{~J} \cdot \mathrm{mol}^{-1} \cdot \mathrm{K}^{-1}\right)$ and $\Delta \mathrm{G}(-26.16$ to -27.69 $\left.\mathrm{kJ} \cdot \mathrm{mol}^{-1}\right)$, indicated that the Hydrogen bonding and van der Waals force played major roles. The binding constant $\left(K_{\mathrm{b}}\right)$ were $4.4545 \times 10^{8} \mathrm{~L} \cdot \mathrm{mol}^{-1}(303 \mathrm{~K})$ and $3.4834 \times 10^{3} \mathrm{~L} \cdot \mathrm{mol}^{-1}(313 \mathrm{~K})$.
\end{abstract}

Keywords-ioscorea nipponica Makino; phageal carcinoma cell; TT; uman serum albumin

\section{INTRODUCTION}

Esophageal carcinoma is the fifth cancer related deaths in men and the eighth in women worldwide. Over the past 15 years, the incidence rate has increased $15 \%{ }^{[1]}$. The incidence is highest in China. Over 500 kinds Chinese traditional herbs have carried out to search effective anti-esophageal carcinoma medicine by our group. The Dioscorea nipponica Makino has been identified as one of the most effective drugs ${ }^{\text {[2] }}$. The Dioscorea nipponica Makino (Japanese Yam) belongs to the Dioscoreaceae, which is widely used on Esophageal cancer. Solvent-refining technology was used to find the active substance of the Dioscorea nipponica Makino. The colourless power (BESDM) obtained by multistep extraction and purification. By MTT assay, the n-butanol fraction showed good cytotoxic activity to the esophageal carcinoma cells (Ec 9706, Eca109 and TE1).

Serum albumin (SA) is the most extensively studied protein, which is the abundant in circulatory system, and have many physiological functions, can transport and dispose many drugs, such as tranquilizers, anesthetics, anti- cancer, anti-coagulants, and a variety of therapeutic drugs ${ }^{[3]}$. The effectiveness of drugs depends on the binding ability between the drugs and SA, the interaction information between drugs and SA can help us understand the absorption, distribution and toxicity of drugs, and the molecular basis of these interactions is indispensable for designing new and more efficient specific therapeutic agents ${ }^{[4]}$. Therefore, it is important to study the interaction between the active ingredient of traditional Chinese medicine with HAS.

In our study, BESDM was extracted from the Dioscorea nipponica Makino as active ingredients. The cytotoxicity of the BESDM on two esophageal carcinoma cells was tested by MTT assay. Under physiological conditions, the interaction mechanism of BESDM with HSA was investigated by spectrofluorimetry. The binding parameters, thermodynamic functions for binding reaction (at $303 \mathrm{~K}$ and $313 \mathrm{~K}$ ) were calculated.

\section{APPARATUS AND REAGENTS}

The main apparatus include $\mathrm{pH} 213$ acidity meter (Portugal) and F-7000 fluorescence spectrophotometer (Hitachi, Japan). Size of the quartz cells was $1.0 \mathrm{~cm}$. The excitation wavelength of HSA studied in this work was 280 nm.

Human serum albumin, HSA (Beijing Olympic nemesis Biological Technology Co. Ltd. China, relative molecular mass 66000), was dissolved daily in $0.1 \mathrm{~mol} / \mathrm{L}$ Tris $-\mathrm{HCl}$ buffer solution $(\mathrm{pH}=7.30)$ to prepare a solution (1×10-5mol/L), Eca109 and TE1 cells (Department of Medical Oncology, Cancer Institute \&Hospital, Chinese Academy of Medical Sciences), Dianion HP-20 (Mitsubishi), silica gel GF254 (BR), BESDM to prepare a solution $(80 \mathrm{mg} / \mathrm{L})$.All chemicals were analytical-reagent grade. All solutions were prepared with double-distilled water. 


\section{RESULTS AND DISCUSSION}

\section{A. Extracting BESDM}

The Dioscorea nipponica Makino were immersed in $95 \%$ ethanol for 5 days at room temperature. Next step was liquid-liquid extraction processes. Crude extraction was dried and suspended in water and successively treated with chloroform, ethyl acetate and n-butanol. By MTT assay, the n-butanol fraction showed good cytotoxic activity to the esophageal carcinoma cells (Ec9706, Eca109 and TE1). Then, the n-butanol fraction subjected to Dianion HP-20 column and successively eluted with $\mathrm{H} 2 \mathrm{O}, 30 \%, 50 \%, 70 \%$, 90\%(v/v) EtOH-H2O, respectively. The 70\% EtOH fraction was chromatographed on silica gel. Sub-fraction was subjected to repeating column chromatography on silica geland, further purified by HPLC and with many times recrystallization, obtained colourless powder (BESDM).

\section{B. Antitumor Activities}

Using the MTT assay, the cytotoxic effect of the BESDM on Ec9706, Eca109 and TE1 have been determined in vitro. The BESDM exhibits inhibition on Eca109 and TE1 cancer cells with IC50 of $7.87 \pm 0.08,3.76 \pm 0.04,13.53 \pm$ $0.06 \mu \mathrm{g} \cdot \mathrm{ml}-1$, respectively. The BESDM has potential application in treatment esophageal carcinoma.

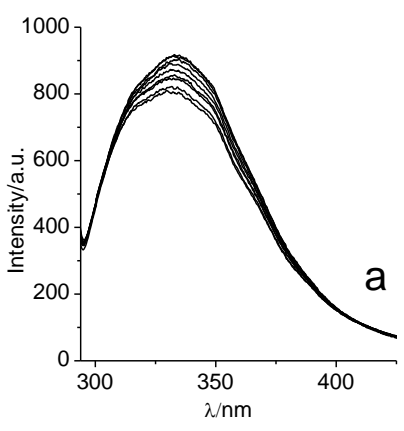

\section{Quenching Fluorescence of HSA}

Fluorescence spectra can gave some information change about the molecular environment of HSA[5]. Fluorescence technique is wide used to research interactions between the active ingredient of traditional Chinese medicine and human serum albumin. HSA has intrinsic fluorescence due to the presence of amino acids, mainly tryptophan (Trp) and tyrosine (Tyr) residues. So, fluorescence technique is wide spread application in investigating of interactions between the active ingredient of Chinese herb and protein molecules [6]. The interaction between BESDM and HSA has been monitored upon exciting HSA at $280 \mathrm{~nm}$ where both Trp and Tyr residues get excited. The variations of emission spectra were shown in Figure 1. The fluorescence quenching spectra of solutions containing a HSA fixed concentration and different concentrations of BESDM at $303 \mathrm{~K}$ and $313 \mathrm{~K}$. Under the experimental condition, the BESDM does not produce any emission in the specified range of study, and there is no significant emission wavelength shift. These results suggest that the interaction between BESDM and HSA occurred, and BESDM can quench the intrinsic fluorescence of HAS.

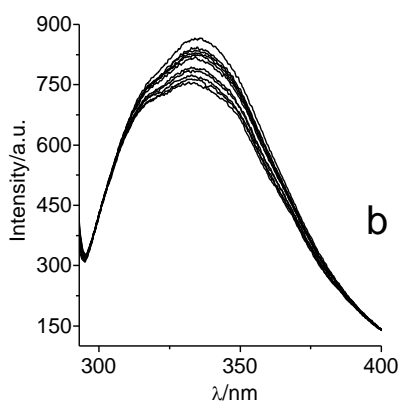

Figure 1. Emission spectra $(\lambda \mathrm{ex}=280 \mathrm{~nm})$ of the HSA $\left(1.0 \times 10^{-5} \mathrm{~mol} / \mathrm{L}\right)$ upon increasing concentrations of BESDM (a) $303 \mathrm{~K}$ and

(b) $313 \mathrm{~K}$

\section{Quenching Mechanism}

There are two quenching types in characterizing the mechanism of the interaction between quenchers and macromolecules: dynamic and static quenching. Dynamic quenching refers to the quencher diffusing towards the fluorophore during the lifetime of the excited state and, upon contact, the fluorophore returns to the ground state without emission of a photon. Static quenching refers to the formation of a non-fluorescence fluorophore-quencher complex. With temperature increasing, the stability of fluorophore-quencher complex reduced, the quenching constant decreased. Dynamic quenching mechanism was probed using the Stern-Volmer equation:

$$
F_{0} / F=1+K_{q} \tau_{0}[\mathrm{Q}]=1+K_{\mathrm{sv}}[\mathrm{Q}]
$$

Where F0 and $\mathrm{F}$ represent the fluorescence intensities of HSA in the absence and in the presence of the external quencher, $\mathrm{Kq}$ is the bimolecular quenching rate constant, KSV is the Stern-Volmer quenching constant, $\tau 0$ is the average lifetime of biopolymers without quencher, the fluorescence lifetime of the biopolymers is $10-8 \mathrm{~s}$, [Q] is the concentration of the quencher.

The plot of $\mathrm{F} 0 / \mathrm{F}$ against ${ }^{[\mathrm{Q}]}$ at different temperature shown in Fig. 2. 

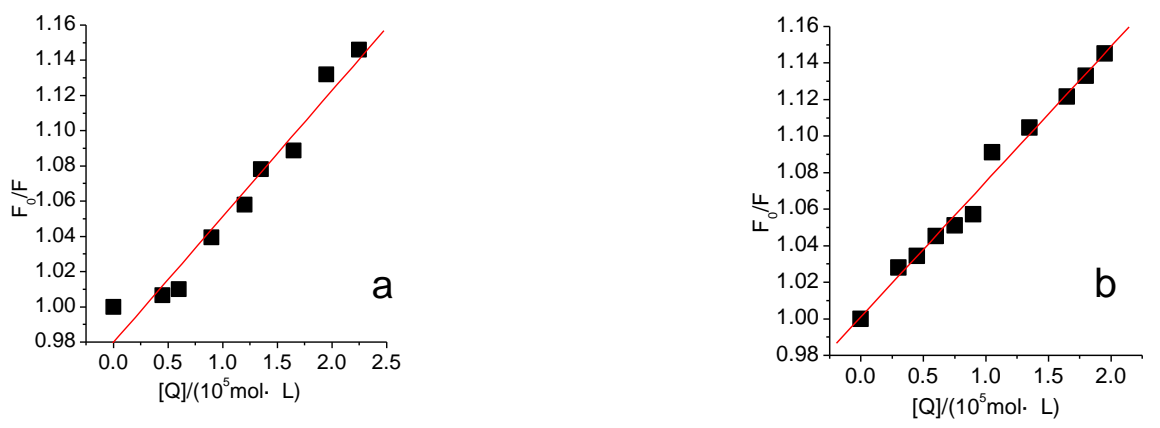

Figure 2. Quenching of HSA by BESDM at (a)303 K and (b) $313 \mathrm{~K}$

Equation (1) was applied to determine KSV by linear regression from a plot of F0/F against [Q], hence, Kq can be caculate by $\mathrm{Kq}=\mathrm{KSV} / \tau 0$. As shown in Table 1 .

TABLE I. CONSTANT OF THE SYSTEMS OF INTERACTION BETWEEN BESDM AND HSA

\begin{tabular}{ccc}
\hline $\mathrm{T} /(\mathrm{K})$ & $K_{\mathrm{sv}} /\left({\left.\mathrm{L} \cdot \mathrm{mol}^{-1}\right)}^{-1}\right)$ & $K_{\mathrm{q}} /\left(\mathrm{L} \cdot \mathrm{mol}^{-1} \cdot \mathrm{s}^{-1}\right)$ \\
\hline 303 & $7.135 \times 10^{3}$ & $7.135 \times 10^{11}$ \\
313 & $7.394 \times 10^{3}$ & $7.394 \times 10^{11}$ \\
\hline
\end{tabular}

For the BESDM-HSA systems, the values of KSV and Kq $=\mathrm{KSV} / \tau 0$ obtained from the plots at $303 \mathrm{~K}$ and $313 \mathrm{~K}$ are shown in Table I. According to the literatures ${ }^{[7,8]}$, which suggest that the fluorescence quenching mechanism is mainly static quenching.

\section{E. Binding Constant and Sites}

The binding constant and the number of binding sites were obtained by the relationship between fluorescence quenching intensity and the concentration of quenchers.

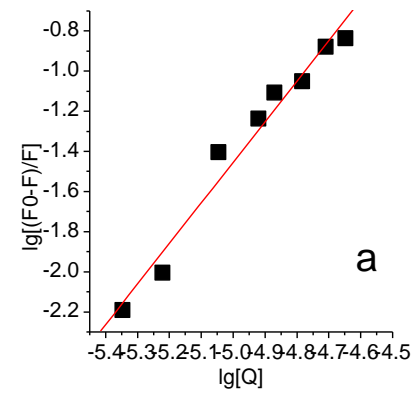

Figure 3. The plots of $\lg (\mathrm{F} 0-\mathrm{F}) / \mathrm{F}$ vs. $\lg [\mathrm{Q}]$ at (a) $303 \mathrm{~K}$ and (b) $313 \mathrm{~K}$
$\operatorname{Lg}(\mathrm{F} 0-\mathrm{F}) / \mathrm{F}=\mathrm{LgKb}+\mathrm{nLg}[\mathrm{Q}]$

At $303 \mathrm{~K}$ and $313 \mathrm{~K}$, the values of $\mathrm{Kb}$ and $\mathrm{n}$ were obtained from the double logarithm regression curve (Figure 3 ). 
TABLE II. THE BINDING CONSTANT, KB, AND THE NUMBER OF BINDING Sites, N

\begin{tabular}{ccccc}
\hline $\mathrm{T} /(\mathrm{K})$ & double logarithm e quation & $\mathrm{R}$ & $K_{\mathrm{b}} /(\mathrm{L} / \mathrm{mol})$ & $\mathrm{n}$ \\
\hline 303 & $\operatorname{Lg}\left[\left(F_{0} / F\right) / F=8.6488+2.021 \mathrm{Lg}[\mathrm{Q}]\right.$ & 0.9851 & $4.4545 \times 10^{8}$ & 2.021 \\
313 & $\operatorname{Lg}\left[\left(F_{0} / F\right) / F=3.5420+0.933 \mathrm{Lg}[\mathrm{Q}]\right.$ & 0.9852 & $3.4834 \times 10^{3}$ & 0.933 \\
\hline
\end{tabular}

The $\mathrm{n}$ values are nearly 2 , and thus indicate the existence of two binding sites in HSA for BESDM. The interactions of BESDM with HSA decreased when rising the temperature, which suggest that interactions of BESDM with HSA were exothermic.

\section{F. Binding modes}

The acting forces between drug and biomolecule are composed of weak interactions of molecules such as hydrogen bond formation, van der Waals forces, electrostatic forces, and hydrophobic interaction. The thermodynamic parameters could be calculated by equation (3), (4) and (5).
$\operatorname{Ln}\left(K_{2} / K_{1}\right)=\Delta \mathrm{rHm}(1 / \mathrm{T} 1-1 / \mathrm{T} 2) / \mathrm{R}$

$\Delta \mathrm{rGm}=-\mathrm{RTLnK}$

$\Delta \mathrm{rSm}=(\Delta \mathrm{rHm}-\Delta \mathrm{rGm}) / \mathrm{T}$

The thermodynamic parameters are presented in Table III.

TABLE III. THERMOdYNAMIC PARAMETERS FOR THE ASSOCIATION OF BESDM With HSA

\begin{tabular}{crcc}
\hline $\mathrm{T} / \mathrm{K}$ & $\Delta \mathrm{rHm} /\left(\mathrm{kJ} \cdot \mathrm{mol}^{-1}\right)$ & $\Delta \mathrm{rSm} /\left(\mathrm{J} . \mathrm{K}^{-1}\right)$ & $\Delta \mathrm{rGm} /\left(\mathrm{kJ} . \mathrm{mol}^{-1}\right)$ \\
\hline 303 & & & -26.16 \\
313 & -1656.79 & -5381.62 & -27.69 \\
\hline
\end{tabular}

As the table shows the values of the $\triangle \mathrm{rHm}, \triangle \mathrm{rSm}$ and $\triangle$ $\mathrm{rGm}$ are all negative numbers. The negative signs reveal that the binding processes are influenced by temperature, low temperature are spontaneous, high temperature are non-spontaneous. The negative $\Delta \mathrm{H}$ and $\Delta \mathrm{S}$ values suggest the van der Waals forces or hydrogen bond formation exist in BESDM and HSA.

\section{CONCLUSION}

By technology of solvent-refining, the active ingredients (BESDM) were extracted from the Dioscorea nipponica Makino. MTT assay was used to test the cytotoxicity of the extracts on esophageal carcinoma cells. The Ic50 values of BESDM on the Ec9706, Eca109 and TE1 are 7.87 \pm 0.08 , $3.76 \pm 0.04$ and $13.53 \pm 0.06 \mu \mathrm{g} \cdot \mathrm{ml}-1$, respectively. The interaction between BESDM and HSA has been investigated under simulated physiological conditions $(\mathrm{pH} 7.3$, ionic strength $0.1 \mathrm{~mol} / \mathrm{L}$ ) using fluorescence methodology. The constant Kq of the BESDM and HSA is larger than $2 \times 1010$ $\mathrm{L} \cdot \mathrm{mol}-1 \cdot \mathrm{s}-1$, which suggest that the fluorescence quenching mechanism of HSA by BESDM was consistent with static quenching. The binding reaction was influenced by temperature for $\Delta \mathrm{rHm}, \Delta \mathrm{rSm}$ and $\Delta \mathrm{rGm}$ is negative. $\Delta \mathrm{H}$ and $\Delta \mathrm{S}$ is negative suggest that Van der Waals forces or hydrogen bond play a major role in the BESDM-HSA interaction.

\section{ACKNOWLEDGMENTS}

This research was supported by the special grade of the financial support from the China Postdoctoral Science Foundation (Grant No.2016T90669), the National Science Foundation for Post-doctoral Scientists of China (Grant No.2015M582189), the National Science Foundation (Grant No.81550014), the Henan Postdoctoral Sustentation Fund, China (Grant No. 00104256), Jinshui District science and technology bureau social program (Grant No. 20132602).

\section{REFERENCES}

[1] Jemal, F. Bray, M. Center, J. Ferlay, E. Ward, D. Forman, Global cancer statistics. CA:A Cancer Journal for Clincians. 61(2011) 69-90.

[2] C. Si, Inhibition of Qigesan and its separated prescriptions on angiogenesis in Esophageal carcinoma Eca109 cell transplanted mude mice tumor. World Chinese Journal of Digestology. 28 (2008)3139-3145.

[3] J. Sankar, D. Sasanka, G. Shalini, G. Nikhil, Binding interaction between plasma protein bovine serum albumin and flexible charge 
transfer fluorophore: A spectroscopic study in combination with molecular docking and molecular dynamics simulation. Journal of Photochemistry and Photobiology A: Chemistry. 231(2012)19-27.

[4] S. M. S. Abdullah, S. Fatma, G. Rabbani, J. M. Ashraf, A spectroscopic and molecular docking approach on the binding of tinzaparin sodium with human serum albumin. Journal of Molecular Structure. 1127 (2017)283-288.

[5] R. Suparna, D.Somnath, P. Chiranjit, Chiranjit.Binding affinities of Schiff base $\mathrm{Fe}$ (II) complex with BSA and calf-thymus DNA: Spectroscopic investigations and molecular docking analysis. Spectrochimica acta. Part A. Spectrochimica acta. Part A, Molecular and biomolecular spectroscopy. 166(2016)84-94.
[6] Cao, D. Wu, H. Wang, M. Xu, Effect of the glycosylation of flavonoids on interaction with protein. Spectrochimica acta. Part A, Molecular and biomolecular spectroscopy. 73(2009) 972-975.

[7] Cui, Y. Yan, Q. Zhang, X. Yao, G. Qu, Y. Lu, Characterization of the interaction between 8-bromoadenosine with human serum albumin and its analytical application. Spectrochimica acta. Part A, Molecular and biomolecular spectroscopy. 74 (2009)964-971.

[8] F. Y. Zhang, Y. N. Ni, A Comparison Study on the Interaction of Sunset Yellow and $\beta$-Carotene with Bovine Serum Albumin Acta Chimica Sinica. 12 (2012)59-64. 